\title{
BMJ Open Effects of a culturally tailored parenting support programme in Somali-born parents' mental health and sense of competence in parenting: a randomised controlled trial
}

\author{
Fatumo Osman, ${ }^{1,2}$ Raziye Salari, ${ }^{3}$ Marie Klingberg-Allvin,, ${ }^{1,2}$ Ulla-Karin Schön, ${ }^{2}$ \\ Renée Flacking ${ }^{2}$
}

To cite: Osman F, Salari $\mathrm{R}$ Klingberg-Allvin $\mathrm{M}$, et al. Effects of a culturally tailored parenting support programme in Somali-born parents' mental health and sense of competence in parenting: a randomised controlled trial. BMJ Open 2017;7:e017600. doi:10.1136/ bmjopen-2017-017600

- Prepublication history for this paper is available online. To view these files, please visit the journal online (http://dx.doi. org/10.1136/bmjopen-2017017600).

Received 3 May 2017

Revised 14 September 2017

Accepted 2 October 2017
CrossMark

'Department of Women's and Children's Health, Karolinska Institutet, Stockholm, Sweden ${ }^{2}$ School of Education, Health and Social Studies, Dalarna University, Falun, Sweden ${ }^{3}$ Department of Public Health and Caring Sciences, ChildHealth and Parenting (CHAP), Uppsala University, Uppsala, Sweden

Correspondence to Fatumo 0sman; fos@du.se

\section{ABSTRACT}

Objectives To evaluate the effectiveness of a culturally tailored parenting support programme on Somali-born parents' mental health and sense of competence in parenting.

Design Randomised controlled trial.

Setting A city in the middle of Sweden.

Participants Somali-born parents $(n=120)$ with children aged 11-16 years and self-perceived stress in their parenting were randomised to an intervention group ( $n=60)$ or a waiting-list control group $(n=60)$.

Intervention Parents in the intervention group received culturally tailored societal information combined with the Connect parenting programme during 12 weeks for 1-2 hours per week. The intervention consisted of a standardised training programme delivered by nine group leaders of Somali background.

Outcome The General Health Questionnaire 12 was used to measure parents' mental health and the Parenting Sense of Competence scale to measure parent satisfaction and efficacy in the parent role. Analysis was conducted using intention-to-treat principles.

Results The results indicated that parents in the intervention group showed significant improvement in mental health compared with the parents in the control group at a 2-month follow-up: $\mathrm{B}=3.62,95 \% \mathrm{Cl} 2.01$ to $5.18, p<0.001$. Further, significant improvement was found for efficacy $(\mathrm{B}=-6.72,95 \% \mathrm{Cl}-8.15$ to -5.28 , $\mathrm{p}<0.001)$ and satisfaction $(\mathrm{B}=-4.48,95 \% \mathrm{Cl}-6.27$ to $-2.69, p<0.001)$ for parents in the intervention group. Parents' satisfaction mediated the intervention effect on parental mental health $(\beta=-0.88,95 \% \mathrm{Cl}-1.84$ to -0.16 , $\mathrm{p}=0.047$ ).

Conclusion The culturally tailored parenting support programme led to improved mental health of Somali-born parents and their sense of competence in parenting 2 months after the intervention. The study underlines the importance of acknowledging immigrant parents' need for societal information in parent support programmes and the importance of delivering these programmes in a culturally sensitive manner.

Clinical trial registration NCT02114593.
Strengths and limitations of this study

- The study design was a randomised controlled trial with a low dropout rate and high retention.

- The culturally tailored parenting support programme was based and constructed on previous qualitative findings.

- The parenting support programme was delivered by group leaders of a similar background to that of the participants

- Data were collected through self-report instruments.

- A limitation is the short interval between the intervention and the follow-up.

\section{INTRODUCTION}

The process of non-voluntary immigration, transitioning and acculturating to a new country may have a negative impact on the mental health of immigrants. ${ }^{1-3}$ Postmigration factors (eg, stress, lack of social capital, social isolation and loss of social network) as well as acculturation problems and experiences of discrimination in the host country affect the mental health of the parents and the children. ${ }^{45}$ Moreover, immigrant parents face challenges concerning their role and responsibilities as parents while adjusting to the host country, all of which tend to create stress in parenting. ${ }^{136}$ The mental health problems of parents have been reported to be a risk factor for children's behavioural problems and may negatively affect the parent-child attachment and their relationship. ${ }^{78}$ Studies have also shown that parents with mental health problems have a low perceived sense of competence in parenting and may lack the ability to employ positive parenting practises. ${ }^{910}$

Studies conducted on different populations have generally demonstrated that parenting support programmes encourage positive 
parenting practices, strengthen parent-child relationships and promote the mental health of parents. ${ }^{11-17}$ Previous studies have linked parenting support programmes with an improvement of parents' sense of competence, ${ }^{18} 19$ which, in turn, has an impact on parents' mental health. ${ }^{20}$ According to Bandura's theory on self-efficacy, stronger self-efficacy in child rearing leads to better satisfaction in parenting and decreased stress and depression. ${ }^{21}$ Some studies have found a positive relationship between parents' sense of competence and parenting behaviour ${ }^{22}$ and that increased maternal self-efficacy is associated with decreased depressive symptoms in postpartum mothers. ${ }^{23}$ To date, it is unclear whether parenting support programmes are effective in improving the mental health of parents directly or via increased self-efficacy and satisfaction in the parenting role.

In addition, little knowledge is available on the effect of parenting support programmes delivered to immigrant parents. ${ }^{24}$ The few studies available have mostly shown little or no improvement in the mental health of immigrant parents ${ }^{2526}$ or even poorer outcomes for immigrant families ${ }^{27}$ and families with low socioeconomic status. ${ }^{28}$ Scarcity of studies in this area may simply because few immigrant parents participate in such programmes. ${ }^{24}$ Several studies have reported difficulties in recruiting and retaining immigrant parents in parenting support programmes. ${ }^{29}{ }^{30}$ Factors such as belonging to an ethnic minority, low socioeconomic status, practical aspects or experienced alienation and discrimination all contribute to low participation. ${ }^{2831}$ Other studies have demonstrated that low participation and a high dropout rate of immigrant parents are associated with a lack of cultural sensitivity in the intervention, poor information about the parenting programme and lack of trust towards professionals. ${ }^{24}$ A qualitative study conducted with Somali-born parents in Sweden showed that Somali parents experienced many societal challenges in the new country and in their parenting behaviours. The parents expressed a need for specific parental support that focuses on parenting in the new country and on strengthening the parent-child relationship. ${ }^{3}$

In a recent randomised controlled trial (RCT), ${ }^{32}$ we showed that an intervention in the form of a culturally tailored parenting support programme was effective in reducing children's behaviour problems 2 months after the intervention, which was our primary outcome measure of the study. In the current paper, we limited our investigation to two of the eight prespecified secondary outcomes with the aim to evaluate the effectiveness of a culturally tailored parenting support programme on the mental health and sense of competence in the parenting of Somali-born parents. Furthermore, we examined whether the intervention affected the mental health of parents, owing to their new sense of competence.

\section{METHODS}

\section{Study design and participants}

The study was designed as an RCT to evaluate the effectiveness of a culturally tailored parenting support programme for Somali-born parents living in Sweden. The trial comprised two arms: parents were randomised to either an intervention group or a waiting-list control group. The study was conducted in a city in the middle of Sweden, of which approximately 3000 of the inhabitants are of Somali origin. Parents were recruited through key persons within Somali associations, social services, schools and a family centre (a meeting place for parents living in the city). All Somali-born parents expressing interest were screened for eligibility. Somali-born parents with children aged 11-16 years and with self-perceived stress related to parenting practices were included in the study. Parents with severe mental illness (eg, psychosis, schizophrenia, bipolar disorder) or participating in another parenting programme were excluded. Eligible parents completed a baseline questionnaire before randomisation and at the 2-month follow-up and were given a gift voucher equivalent to $\mathrm{Kr} 150$ (Swedish Kroner) (approximately US\$15). Ethical approval for the study was obtained from the Swedish Regional Ethical Review Board in Uppsala, Sweden (Dnr 2014/048). All participants gave both oral and written informed consent.

\section{Intervention}

The parenting intervention consisted of 12 group-based sessions lasting on average about 1-2 hours, combining culturally tailored societal information with the Connect parenting support programme, which has been described elsewhere. ${ }^{33}$ The first two sessions were designed based on results from earlier findings on qualitative focus group discussions. ${ }^{3}$ The aim of the culturally tailored societal information aspect of the intervention was to give Somali-born parents an introduction on parenting styles, the rights of the child, the family legal system in relation to parenting and the goal of the work of social services with children and family. The other 10 sessions constituted the Connect parenting support programme. The Connect is a standardised programme based on attachment theory and focuses on strengthening the parent-child relationship and attachment. The content aims to enhance and stimulate parents to reflect on how they respond to their child's behaviours and to build a trusting and secure relationship. ${ }^{33}$ The Connect programme was adapted and modified in relation to role play and examples to make it understandable for the participants without changing the programme's core components. In total, nine group leaders (five male and four female) of Somali background delivered the intervention. Each session of the Connect programme was administered by two group leaders (one male and one female) together with sex-mixed groups of 12-17 parents. The intervention was held near the participants' neighbourhood. Participants were offered child care services during the sessions and the possibility for support (eg, in reading letters from the municipality or migration office). 


\section{Enrolment}
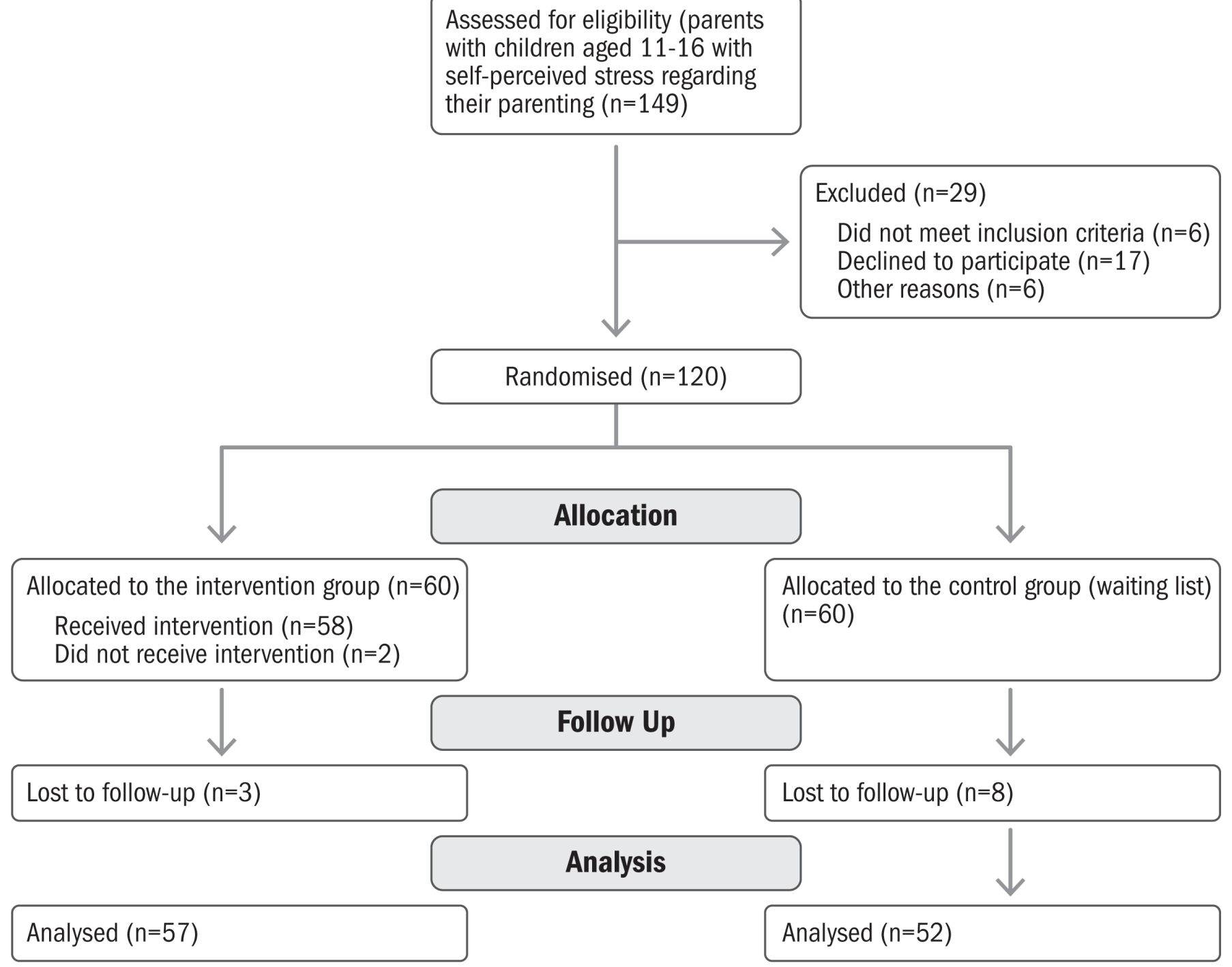

Figure 1 Participant flow chart.

\section{Outcome measures}

The main outcome measure was reduced emotional and behavioural problems in children. ${ }^{32}$ Secondary outcomes were improved mental health of the parents and sense of competence in parenting.

The General Health Questionnaire 12 (GHQ-12) $^{34}$ is a 12-item version of the original GHQ and measures parents' mental health. The GHQ is a psychometric self-administered screening device to measure psychiatric distress experienced by an individual over the past few weeks. Parents answered each item on a four-point Likert scale ranging from 1 (eg, better than usual) to 4 (eg, much less than usual), with higher scores indicating higher mental health distress. A total score is calculated by summing up all the items (total scores can range from 12 to 48$).^{34}$
The Parenting Sense of Competence (PSOC) scale ${ }^{35}$ was used to measure the participating parents' sense of competence in parenting. The PSOC comprises 16 items divided into two subscales (satisfaction with nine items and efficacy with seven items). Parents responded on a six-point Likert scale anchored at $1=$ strongly disagree and $6=$ strongly agree. The total score ranged from 9 to 54 for the satisfaction items and 7 to 42 for the efficacy items. The satisfaction items were reverse coded; a higher score in both satisfaction and efficacy subscales indicates a higher parent sense of competence. ${ }^{35}$

Participants were also asked about their sociodemographic background (eg, age, sex, marital status, education level, number of years in Sweden, employment status, residential area, visits to cultural and community events, financial situation, number of children, children's age 
Table 1 Participant characteristics at baseline (intervention group $n=60$, control group $n=60$ )

\begin{tabular}{|c|c|c|c|c|}
\hline \multirow[t]{2}{*}{ Variable } & \multicolumn{2}{|c|}{ Intervention group } & \multicolumn{2}{|c|}{ Control group } \\
\hline & $\mathbf{n}$ & $\%$ & $\mathbf{n}$ & $\%$ \\
\hline \multicolumn{5}{|l|}{ Participants (parents) } \\
\hline Mothers & 43 & 72 & 37 & 62 \\
\hline Fathers & 17 & 28 & 23 & 38 \\
\hline Participants' age, years (mean \pm SD) & $44 \pm 8$ & & $45 \pm 9$ & \\
\hline \multicolumn{5}{|l|}{ Years in Sweden } \\
\hline $1-5$ years & 39 & 65 & 34 & 57 \\
\hline $6-9$ years & 10 & 17 & 19 & 32 \\
\hline$\geq 10$ years & 11 & 18 & 7 & 12 \\
\hline \multicolumn{5}{|l|}{ Highest educational level } \\
\hline <upper secondary school & 37 & 62 & 32 & 54 \\
\hline Upper secondary school & 22 & 37 & 22 & 37 \\
\hline Higher education & 1 & 2 & 5 & 9 \\
\hline \multicolumn{5}{|l|}{ Occupation } \\
\hline Unemployed & 13 & 22 & 11 & 19 \\
\hline Parental leave & 13 & 22 & 6 & 10 \\
\hline Studying & 29 & 48 & 31 & 53 \\
\hline Employed & 5 & 8 & 11 & 19 \\
\hline \multicolumn{5}{|l|}{ Civic status } \\
\hline Single & 21 & 35 & 18 & 30 \\
\hline Married & 39 & 65 & 41 & 70 \\
\hline Cohabiting with partner & 31 & 52 & 34 & 57 \\
\hline No of children living at home $($ mean $\pm S D)$ & $5 \pm 2$ & & $5 \pm 3$ & \\
\hline Concerns about their financial situation & 21 & 36 & 15 & 26 \\
\hline Child's sex: male & 36 & 60 & 33 & 55 \\
\hline Child's age, years $($ mean $\pm S D)$ & $14 \pm 2$ & & $13 \pm 2$ & \\
\hline \multicolumn{5}{|l|}{ Mental health } \\
\hline GHQ $12($ mean \pm SD $)$ & 20.0 & & 19.71 & \\
\hline \multicolumn{5}{|l|}{ PSOC } \\
\hline Efficacy $(m e a n \pm S D)$ & 17.9 & & 18.66 & \\
\hline Satisfaction (mean \pm SD) & 31.5 & & 30.77 & \\
\hline
\end{tabular}

GHQ, General Health Questionnaire; PSOC, Parenting Sense of Competence scale.

and sex). Both instruments (the GHQ-12 and the PSOC) were translated according to international guidelines. ${ }^{36} 37$ Approval to translate and use the GHQ-12 was obtained from instrument developers.

\section{Sample size}

Sample size was calculated based on the primary outcome, that is, reduced emotional and behavioural problems in the children with a medium effect size (Cohen's $d=0.5$ ). The findings of the primary outcome measure have been published elsewhere. ${ }^{32}$ A sample of 128 parents/children ( $\mathrm{n}=64$ in the intervention group, $\mathrm{n}=64$ in the control group) were required ${ }^{27}$ with alpha set at $\mathrm{p}<0.05$ and power at 0.80 .

\section{Randomisation}

The randomisation list was prepared using a computer sequence generator programme with permutated blocks to determine sequence numbers for allocation to the intervention and wait-list control group. Block randomisation, using blocks of 10, was done to obtain an equal distribution. Group affiliation and study number were noted on a piece of paper and placed in a set of identical opaque envelopes by the first author (FO). The envelopes were then sealed and shuffled. Thus, this procedure ensured that the content of each envelope was not known to either the researchers or the participants.

Randomisation was performed after the baseline data were collected by the first author and research 
Table 2 ANCOVA on changes in parent outcomes with effect size estimates at the 2 month follow-up

\begin{tabular}{|c|c|c|c|c|c|}
\hline & Intervention group $(n=57)$ & Control group $(n=52)$ & $\begin{array}{l}\text { Model-based mean } \\
\text { difference }\end{array}$ & p Value & Effect size \\
\hline & Follow-up (mean $\pm S D$ ) & Follow-up (mean $\pm S D$ ) & B (95\% Cl) & & Cohen's d \\
\hline \multicolumn{6}{|l|}{ Mental health problems } \\
\hline GHQ 12 & $17.68 \pm 4.57$ & $21.13 \pm 4.16$ & 3.62 (2.01 to 5.18$)$ & $<0.001$ & 0.85 \\
\hline \multicolumn{6}{|l|}{ Mediators } \\
\hline PSOC, efficacy & $28.53 \pm 4.50$ & $21.79 \pm 2.69$ & $-6.72(-8.15$ to -5.29$)$ & $<0.001$ & 1.81 \\
\hline PSOC, satisfaction & $26.63 \pm 5.80$ & $22.10 \pm 2.95$ & $-4.48(-6.27$ to -2.69$)$ & $<0.001$ & 0.98 \\
\hline
\end{tabular}

Low scores in mean $\mathrm{GHQ}=$ reduced mental health problems.

Higher scores in mean PSOC=higher efficacy and satisfaction.

Cohen's d estimates the effect size of parent outcome at the 2 -month follow-up (small effect $d=0.2$, medium effect $d=0.5$, large effect $d=0.8$, very large effect $d=1.45$ ).

ANCOVA, analysis of covariance; GHQ, General Health Questionnaire; PSOC, Parenting Sense of Competence scale.

assistants. After each participant completed the questionnaire, the individual chose one opaque sealed envelope and at that time was informed whether he or she was allocated to the intervention or control group. Participants allocated to the control group were informed that they would receive the intervention once all data had been collected from both groups. After the parents in the intervention group had completed the intervention, a 2-month follow-up was conducted for both intervention and control participants. Only data from one parent per family (the parent who was screened and gave written informed consent) was used in the event both parents participated in the intervention sessions. The researchers were not blinded to group assignment.

\section{Statistical methods}

An intention-to-treat analysis was conducted which included all randomised participants in the groups to which they were randomly assigned, regardless of the number of sessions in which they participated, if data were available for follow-up. The effectiveness of the randomisation procedure was validated by comparing the intervention and control group at baseline using a series of $\chi^{2}$ and t-tests. The analysis started by reconstructing the scale of the GHQ-12 and the two subscales of the PSOC. There were a few cases of missing data $(0.42 \%$ in the GHQ and $1.3 \%$ in the PSOC) because some participants failed to answer all the items. If a participant had missed $<30 \%$ of the items on a particular scale, we constructed the scale by imputing the mean of the scale for the missing items. Because all of the participants who had been followed up (109 cases) had completed at least $70 \%$ of the items on each scale, this resulted in the retention of the full sample in all the analyses.

An analysis of covariance (ANCOVA) was performed to study the intervention effects on the dependent variables (ie, the GHQ items and the two subscales of the PSOC) by examining differences between the intervention and control group at follow-up, controlling for baseline measures. Cohen's d effect sizes were calculated, with $\mathrm{d}=0.2$ regarded as small effect, $\mathrm{d}=0.5$ as a medium effect, $\mathrm{d}=0.8$ as a large effect and $\mathrm{d}=1.45$ as a very large effect. ${ }^{38}$

To determine whether the intervention led to a clinically meaningful and reliable change the reliable change index was computed, as recommended by Jacobson and Truax. ${ }^{39}$ Because population norms for the GHQ-12 and PSOC were not available for the present study population, we calculated the SE of difference $\left(\mathrm{S}_{\text {diff }}\right)$ based on the pretest scores for the intervention and control group combined, assuming a measurement reliability of 0.8 for each measure. The clinical significance of change from

Table 3 Clinical significance of the intervention effects: proportions of scores showing reliable change

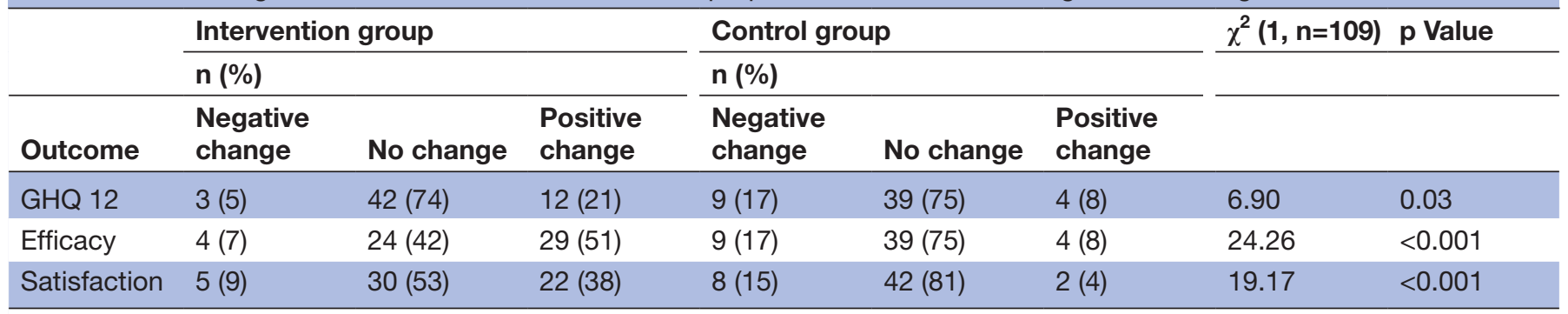

GHQ, General Health Questionnaire. 


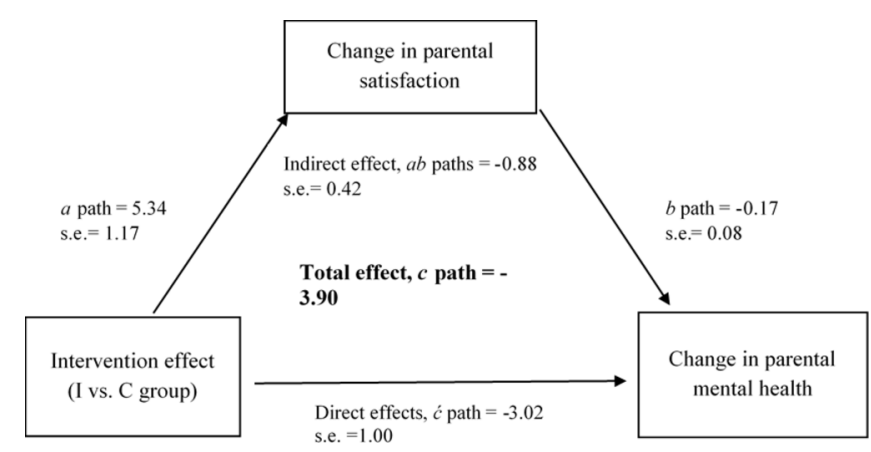

Figure 2 Simple mediation model of the intervention effect on change in parental mental health accounting for the mediator, that is, parental satisfaction. Path coefficient, standardised $\beta s=$ adjusted mean estimate. Direct effect=direct effect of the intervention on change in parental mental health. Indirect effect=total effect-direct effect. Total effect=direct effect+indirect effect.

baseline to the 2-month follow-up was then tested with $\chi^{2}$ tests by comparing the proportion of parents in the intervention and control group who had deteriorated, remained unchanged or improved in mental health as well as in efficacy and satisfaction.

A stepwise approach was taken to identify which independent variables (ie, parental efficacy or parental satisfaction) should be included in the mediation model. In the first step, a regression analysis was conducted with change in mental health as the dependent variable and group membership (intervention or control group), parental satisfaction and efficacy as the independent variables. In this regression, only parental satisfaction emerged as a significant predictor of change in parental mental health and was therefore included in the mediation analysis in the next step. Mediator analyses were performed following the suggestion of Hayes. ${ }^{39}$ In the first step, we tested whether the intervention predicted decreased mental health problems (direct effect, $c$ path). In the second step, we examined the intervention effect on the mediator, that is, parental satisfaction ( $a$ path). In the third step, we tested whether the mediator was related to the outcome (ie, change in mental health) after the group assignment was controlled ( $b$ path). In the fourth step, we assessed the indirect effect of the intervention on outcome (ie, change in mental health). Finally, the total effect of the intervention was examined. The analyses were conducted using SPSS V.21 (IBM). The mediation analyses were performed using SPSS macro developed by Preacher and Hayes, ${ }^{40}$ which calculates total, direct and indirect effects, including bootstrap procedures to calculate CIs. We used a resample procedure of 10000 bootstrap samples (bias corrected and accelerated estimates and $95 \%$ CIs).

\section{RESULTS}

The study started May 2014 and ended in October 2015. In total, 149 parents were assessed for study eligibility and 120 parents were randomly assigned to the intervention group ( $\mathrm{n}=60$ parents) and the control group ( $\mathrm{n}=60$ parents). Of these 120 parents, $109(90 \%)$ were successfully followed-up (57 in the intervention group and 52 in the control group). Of the 60 parents randomised to the intervention group, two did not attend any session and these could not be reached for follow-up. Overall, $70 \%$ of the parents $(n=80)$ attended more than eight sessions. Few participants $(30 \%)$ opted to use the child care services and support system (eg, to have the child care services read letters from the municipality during the 12 group-based sessions). The participation flowchart of each group is represented in figure 1.

\section{Participant characteristics at baseline}

Table 1 presents the sociodemographic background of the respondents. There were no differences between the intervention and control groups in sociodemographic background. Most of the parents $(98.3 \%, \mathrm{n}=118)$ were biological parents of the child in the study. Of the parents who participated in the study, the majority had lived in Sweden between 1 and 5 years, had less than an upper secondary level of education and lived in a low socioeconomic status area.

There were no significant differences between the two groups or between fathers and mothers in financial strain, or baseline measures of mental health, efficacy and satisfaction in their parenting.

\section{Effects of the intervention on parents' mental health and sense of competence}

The ANCOVA analyses (table 2) indicated that the parents in the intervention group had improved their mental health more than the parents in the control group 2 months after the intervention (95\% CI, 2.02 to 5.18). The associated effect size was large (Cohen's $\mathrm{d}=0.85$ ). Similarly, the intervention had a positive effect on parents' sense of competence in parenting. Parents in the intervention group reported greater improvements in both their parenting efficacy $(95 \% \mathrm{CI}-8.15$ to -5.29 ; $\mathrm{d}=1.79)$ and satisfaction $(95 \% \mathrm{CI}-4.48$ to $-2.69 ; \mathrm{d}=0.89)$ compared with parents in the control group.

\section{Clinical significance change}

Table 3 shows the results from the clinical significance analysis. Although most parents remained unchanged, 12 parents $(21 \%)$ in the intervention group demonstrated reliable improvement (measured by the GHQ-12) compared with only four (8\%) in the control group. The positive changes were more pronounced for sense of competence in parenting with $29(51 \%)$ parents in the intervention group showing reliable improvement in parenting efficacy and $22(38 \%)$ showing improvement in parental satisfaction. Corresponding figures in the control group were four (8\%) parents showing improvement in parenting efficacy and two (4\%) showing improvement in parental satisfaction. 


\section{Mediation model}

The mediation analysis (figure 2) demonstrated a significant direct relation between the intervention and change in parental mental health ( $c$ path, $\beta=-3.02, \mathrm{p}=0.003$ ). In addition, the intervention had a positive association with parental satisfaction ( $a$ path, $\beta=5.34, \mathrm{p}<0.001$ ). In turn, parental satisfaction had a significant relation with change in parental mental health ( $b$ path) when group assignment was controlled $(\beta=-0.17, p=0.03)$. When the intervention effect and parental satisfaction were entered simultaneously in the last regression, a significant indirect effect ( $a b$ paths) was found from the intervention effect to change in parental mental health through parental satisfaction $(\beta=-0.88,95 \%$ CI -1.84 to $-0.16, p=0.047)$, indicating that the intervention effect on parental mental health was partially mediated by parental satisfaction. Finally, the total effect of change on parents' mental health ( $c$ path) was significant $(\beta=-3.90, \mathrm{p}<0.001)$, indicating that parents who received the intervention had improved mental health. The model explained $16 \%\left(\mathrm{R}^{2}\right.$ $0.16, \mathrm{p}<0.001)$ of the change in parents' mental health.

\section{DISCUSSION}

Our study shows that a culturally tailored parenting support programme improved the mental health and sense of competence in parenting of Somali-born parents 2 months after the intervention. These improvements were both statistically significant and clinically meaningful. The findings also indicate that parental satisfaction was a mediating factor in parents' mental health.

Our findings are consistent with findings of earlier that show parenting programmes are generally effective in improving parents' mental health ${ }^{14}$ but disagrees with some other studies in which parenting support programmes for immigrant parents did not have positive effects on parents' mental health. ${ }^{2526}$ For example, a trial conducted on immigrant mothers from Pakistan and Somalia $^{25}$ showed that the parenting support programme was not effective in alleviating maternal mental distress. The most likely explanation for the positive effect is that the culturally tailored societal information addressed an important need for Somali-born parents. Previous studies $\begin{array}{llll}1 & 6 & \text { have reported that immigrant parents }\end{array}$ encounter obstacles in their parenting in the host country (eg, insufficient information about the parenting system, role change and power conflict between parents and children, all of which contribute to stress in parenting). A second possible explanation is that the parenting intervention was culturally tailored (eg, the role plays and reflection exercises in the Connect programme). These role plays and reflection exercises were made more culturally understandable by using metaphors and proverbs (the Somali culture is in part characterised by oral tradition of poetry and narrative). ${ }^{41}$ Using the metaphors and proverbs can have a powerful impact on learning and understanding when employing complex or theoretical terms. A third explanation is that the group leaders who delivered the intervention had a similar background as the participating parents and were therefore 'culturally tailored' to the parents. Several studies have underlined the importance of finding ways to retain ethnic minorities and immigrants and to make the parenting programmes more attractive and effective. ${ }^{1142-44}$ The group leaders were bilingual and were familiar with both Somali and Swedish cultures, which were strengths as nothing was 'lost in translation'. A trial from Norway ${ }^{25}$ and a meta-analytic review ${ }^{24}$ suggest that parenting support programmes appear to be more effective when they are tailored to the specific challenges and needs of immigrant parents (ie, delivered to participants in their own language and by group leaders of a similar background). A final possible explanation is the focus of the parenting programme Connect, ${ }^{45}$ which encourages parents to reflect on their parenting role and develop sensitivity towards their children's behaviour. Parents are taught to think and better understand the reason behind the child's emotional reactions and to develop awareness on how to respond in a way that acknowledges the child's attachment needs. Our qualitative study shows that parents requested support to strengthen the relationship with their children in the new host country. ${ }^{3}$

Our findings demonstrate that parents' sense of competence in parenting improved with a large effect size $(d=0.89)$ in parental satisfaction and a huge effect size $(d=1.79)$ in parental self-efficacy. Additionally, parental satisfaction mediated the intervention effect on change in parental mental health. Strong feelings of self-efficacy and satisfaction in parenting lead to positive mental health and parenting practices. ${ }^{20-2346}$ Studies have suggested that immigrant parents who encounter challenges in acculturating within the host environment experience stress in parenting, ${ }^{136}$ which is an ample reason to feel a lower level of sense of competence in parenting and in mental health. ${ }^{46}$ Satisfaction in parenting is one factor among others that impact parents' mental health. The mental health of parents is affected by other factors as well, including acculturation, social capital, social isolation and experiencing discrimination because of race or ethnicity. ${ }^{45}$ However, we hypothesise that with increased parental satisfaction, parents gain greater optimism in their parenting, which, in turn, affects their mental health as confirmed by a recent Swedish study. ${ }^{19}$

From a clinical and practical standpoint, it is important to acknowledge the extent to which the intervention improved parents' mental health and sense of competence in parenting. According to Jacobson and Truax, ${ }^{39}$ statistically significant and large effect sizes do not necessarily translate into clinically meaningful changes (ie, an intervention effect may be statistically significant but clinically trivial). The results of the reliable change analyses indicate that the intervention had indeed led to clinically meaningful changes in parental mental health and in a sense of competence in parenting.

There are several strengths and limitations to this study. One of the strengths is our use of an RCT research design 
to reduce selection bias and spurious causality inferences. Another strength was the low dropout rate and that we retained almost all parents $(90 \%)$ at the 2-month follow-up. Furthermore, two-thirds of the parents attended more than eight sessions. A contributing factor to the low dropout rate and high rate of participation was the involvement of civil society (such as key people within Somali associations and having different information meetings about the research project). Furthermore, the group sessions were led by group leaders of Somali background who shared the same language and culture as the parents. One limitation is the short interval between the intervention and the follow-up. Another limitation is that the data were collected using a self-report measure. This study can be generalised to Somali-born parents who have experienced war or social conflict and stress in parenting, and the cultural sensitive model in this study can be applied and generalised to hard-to-reach groups.

\section{Conclusions and implications for clinical practice}

This study found that culturally tailored parenting support programme improved the mental health and sense of competence in parenting in Somali-born adults, with large effect sizes 2 months after the intervention ended. Our study highlights the importance of acknowledging immigrant parents' need for societal information in parent support programmes and that these programmes must be delivered in a culturally sensitive way. Improving the parents' mental health and sense of competence in parenting is associated with a positive effect on children's behavioural problems and the parent-child relationship, which promotes equity in health. The current study shows that a culturally tailored programme can be offered to all parents with self-perceived parenting-related stress, regardless of whether their children have emotional or behavioural problems or not. These findings underscore the beneficial effects of making culturally tailored parenting programmes accessible to immigrant parents.

\section{ACKNOWLEDGEMENTS}

The authors would like to thank all the parents who participated in this study, including Somali non-profit associations (eg, UMIS) and key persons who assisted with the information meetings and recruitment process. We also would like to thank all the group leaders who delivered the intervention and the data collectors for assisting in data collection. We thank the Public Health Agency of Sweden for funding this study.

Contributors FO, MK-A, U-KS and RF conceptualised and designed the study and directed the planning and implementation of the trial. F0 collected the data. RS and FO were responsible for data analyses and interpretation in which RF contributed to interpretation of the results. F0 produced the draft manuscript to which all authors contributed and provided feedback during its development. All authors approved the final manuscript as submitted.

Funding This work was funded by the Public Health Agency of Sweden, grant number: 802/2014-6.2.

Competing interests None declared.
Ethics approval Swedish Regional Ethical Review Board in Uppsala.

Provenance and peer review Not commissioned; externally peer reviewed.

Data sharing statement Data sharing on request after assessment from the research group and ethical approval.

Open Access This is an Open Access article distributed in accordance with the Creative Commons Attribution Non Commercial (CC BY-NC 4.0) license, which permits others to distribute, remix, adapt, build upon this work non-commercially, and license their derivative works on different terms, provided the original work is properly cited and the use is non-commercial. See: http://creativecommons.org/ licenses/by-nc/4.0/

(c) Article author(s) (or their employer(s) unless otherwise stated in the text of the article) 2017. All rights reserved. No commercial use is permitted unless otherwise expressly granted.

\section{REFERENCES}

1. Heger Boyle E, Ali A. Culture, Structure, and the Refugee Experience in Somali Immigrant Family Transformation. Int Migr 2010;48:47-79.

2. Lewig K, Arney F, Salveron M. Challenges to parenting in a new culture: Implications for child and family welfare. Eval Program Plann 2010;33:324-32.

3. Osman F, Klingberg-Allvin M, Flacking R, et al. Parenthood in transition-Somali-born parents' experiences of and needs for parenting support programmes. BMC Int Health Hum Rights 2016;16:1-11.

4. Grant J, Guerin PB. Applying ecological modeling to parenting for Australian refugee families. J Transcult Nurs 2014;25:325-33.

5. Missinne S, Bracke P. Depressive symptoms among immigrants and ethnic minorities: a population based study in 23 European countries. Soc Psychiatry Psychiatr Epidemiol 2012;47:97-109.

6. Filio DS, Mulki P. Somali parents' experiences of bringing up children in Finland: exploring social-cultural change within migrant households.. Forum Qual Soc Res. In Press. 2006;7.

7. Cummings EM, George MR, Koss KJ, et al. Parental depressive symptoms and adolescent adjustment: responses to children's distress and representations of attachment as explanatory mechanisms. Parent Sci Pract 2013;13:213-32.

8. Kane P, Garber J. Parental depression and child externalizing and internalizing symptoms: Unique effects of fathers' symptoms and perceived conflict as a mediator. J Child Fam Stud 2009;18:465-72.

9. Dix T, Meunier LN. Depressive symptoms and parenting competence: an analysis of 13 regulatory processes. Dev Rev 2009;29:45-68.

10. Gelkopf M, Jabotaro SE. Parenting style, competence, social network and attachment in mothers with mental illness. Child Fam Soc Work 2013;18:496-503.

11. Stewart-Brown SL, Schrader-McMillan A. Parenting for mental health: what does the evidence say we need to do? Report of workpackage 2 of the dataprev project. Health Promot Int 2011;26 Suppl 1:i10-i28.

12. Stewart-Brown S, Patterson J, Mockford C, et al. Impact of a general practice based group parenting programme: quantitative and qualitative results from a controlled trial at 12 months. Arch Dis Child 2004;89:519-25.

13. Morgan Z, Brugha T, Fryers T, et al. The effects of parent-child relationships on later life mental health status in two national birth cohorts. Soc Psychiatry Psychiatr Epidemiol 2012;47:1707-15.

14. Barlow J, Smailagic N, Huband N, et al. Group-based parent training programmes for improving parental psychosocial health. Cochrane Database of Syst Rev 2012:CD002020.

15. Stattin $H$, Enebrink $P$, Özdemir $M$, et al. A national evaluation of parenting programs in Sweden: The short-term effects using an RCT effectiveness design. J Consult Clin Psychol 2015;83:1069-84.

16. Stewart-Brown S. Improving parenting: the why and the how. Arch Dis Child 2008;93:102-4.

17. Coleman PK, Karraker KH. Self-Efficacy and Parenting Quality: Findings and Future Applications. Developmental Review 1998;18:47-85.

18. Sanders MR, Kirby JN, Tellegen CL, et al. The Triple P-Positive Parenting Program: a systematic review and meta-analysis of a multilevel system of parenting support. Clin Psychol Rev 2014;34:658.

19. Löfgren $\mathrm{HO}$, Petersen $\mathrm{S}$, Nilsson $\mathrm{K}$, et al. Effects of parent training programmes on parents' sense of competence in a general population sample. Glob J Health Sci 2017;9:24-34.

20. Pisterman S, Firestone P, McGrath P, et al. The effects of parent training on parenting stress and sense of competence. Can J Behav Sci 1992;24:41-58. 
21. Bandura, A. S-efficacy. Ramachaudran VSEncyclopedia of human behavior. , ed. Encyclopedia of mental health. San Diego: New York: Academic Press, 1998:71-81.

22. de Haan AD, Prinzie P, Deković M. Mothers' and fathers' personality and parenting: the mediating role of sense of competence. Dev Psychol 2009;45:1695-707.

23. Haslam DM, Pakenham KI, Smith A. Social support and postpartum depressive symptomatology: The mediating role of maternal selfefficacy. Infant Ment Health J 2006;27:276-91.

24. Griner D, Smith TB. Culturally adapted mental health intervention: a meta-analytic review. Psychotherapy 2006;43:531-48.

25. Bjørknes R, Larsen M, Gwanzura-Ottemöller F, et al. Exploring mental distress among immigrant mothers participating in parent training. Child Youth Serv Rev 2015;51:10-17.

26. Leijten $\mathrm{P}$, Raaijmakers MA, Orobio de Castro B, et al. Effectiveness of the incredible years parenting program for families with socioeconomically disadvantaged and ethnic minority backgrounds. J Clin Child Adolesc Psychol 2017;46:59-73.

27. van Mourik K, Crone MR, Pels TVM, et al. Parents' beliefs about the cause of parenting problems and relevance of parenting support: Understanding low participation of ethnic minority and low socioeconomic status families in the Netherlands. Child Youth Serv Rev 2016:61:345-52.

28. Reyno SM, McGrath PJ. Predictors of parent training efficacy for child externalizing behavior problems--a meta-analytic review. $J$ Child Psychol Psychiatry 2006;47:99-111.

29. Baker CN, Arnold DH, Meagher S. Enrollment and attendance in a parent training prevention program for conduct problems. Prev Sci 2011;12:126-38.

30. Kazdin AE, Holland L, Crowley M. Family experience of barriers to treatment and premature termination from child therapy. $J$ Consult Clin Psychol 1997;65:453-63.

31. Koerting J, Smith E, Knowles MM, et al. Barriers to, and facilitators of, parenting programmes for childhood behaviour problems: a qualitative synthesis of studies of parents' and professionals' perceptions. Eur Child Adolesc Psychiatry 2013;22:653-70.

32. Osman F, Flacking R, Schön UK, et al. A support program for Somali-born parents on children's behavioral problems. Pediatrics 2017;139:e20162764.
33. Moretti MM, Braber K, Obsuth I. Connect. An attachment-focused treatment group for parents and caregivers: a principle based manual. Burnaby, British Columbia, Canada: Simon Fraser University, 2009.

34. Goldberg DP, Gater R, Sartorius N, et al. The validity of two versions of the GHQ in the WHO study of mental illness in general health care. Psychol Med 1997;27:191-7.

35. Gilmore L, Cuskelly M. Factor structure of the parenting sense of competence scale using a normative sample. Child Care Health Dev 2009;35:48-55

36. Institute, M.R. Linguistic validation of a patient reported outcomes measure. Lyon, France: Mapi Research Institute, 2005.

37. World Health Organization. Process of translation and adaptation of instruments: World Health Organazation. http://www.who.int/ substance_abuse/research_tools/translation/en/. (accessed 5 Feb 2016).

38. Cohen J. A power primer. Psychol Bull 1992;112:155-9.

39. Jacobson NS, Truax P. Clinical significance: a statistical approach to defining meaningful change in psychotherapy research. J Consult Clin Psychol 1991;59:12-19.

40. Preacher KJ, Hayes AF. Asymptotic and resampling strategies for assessing and comparing indirect effects in multiple mediator models. Behav Res Methods 2008;40:879-91.

41. Andrzejewski BW, Andrzejewski S. An anthology of Somali poetry. Indiana Univ Pr 1993.

42. Bjørknes R, Jakobsen R, Nærde A. Recruiting ethnic minority groups to evidence-based parent training. Who will come and how? Child Youth Serv Rev 2011;33:351-7.

43. Ortiz C, Del Vecchio T. Cultural diversity: do we need a new wake-up call for parent training? Behav Ther 2013;44:443-58.

44. Wells MB, Sarkadi A, Salari R. Mothers' and fathers' attendance in a community-based universally offered parenting program in Sweden. Scand J Public Health 2016;44:274-80.

45. Moretti MM, Obsuth I, Craig SG, et al. An attachment-based intervention for parents of adolescents at risk: mechanisms of change. Attach Hum Dev 2015;17:119-35.

46. Costigan $\mathrm{C}$, Su TF. Cultural predictors of the parenting cognitions of immigrant Chinese mothers and fathers in Canada. Int $J$ Behav Dev 2008;32:432-42. 